\title{
Extraction of Maxillary Teeth Using 4\% Articaine with Buccal Infiltration Only in Comparison with 2\% Lidocaine Buccal and Palatal Infiltration
}

\section{Hussam Omer Ahmed Mohamed El-Amin ${ }^{1 *}$ and Elneel Ahmed MohamedAli'}

${ }^{1}$ Specialist of Oral and Maxillofacial Surgery BDS, MSc (OMFS), Department Of Oral and Maxillofacial Surgery, Faculty of Dentistry, University Of Khartoum

${ }^{2}$ Associate Professor and Senior Consultant of Oral and Maxillofacial Surgery BDS, M.Sc (OMFS), FSMSB

*Corresponding Author: Hussam Specialist of Oral and Maxillofacial Surgery BDS, MSc (OMFS), Department Of Oral and Maxillofacial Surgery, Faculty Of Dentistry, University Of Khartoum.
Received: September 09, 2021

Published: September 25, 2021

(C) All rights are reserved by Hussam Omer

Ahmed Mohamed El-Amin and Elneel

Ahmed MohamedAli.

\begin{abstract}
Background: 4\% Articaine is a local anaesthetic agent that has a more superior anaesthetic effect compared to lidocaine.

Study design: A quasi experimental study.

Setting: Khartoum teaching dental hospital (KTDH).

Objective: To evaluate the efficiency of articaine as a local anesthetic agent when extracting maxillary teeth using buccal infiltration only compared to Lidocaine buccal and palatal infiltration.

Methods: Patients with maxillary bilateral extraction were injected using a Dental syringe with a short needle (infiltration) on one side with lidocaine $2 \%$ buccally and palatally (control) and on another appointment the other side was injected with $4 \%$ articaine on the buccal side only (infiltration) using a Dental syringe with a short needle. Visual analogue scale (VAS) was used to assess the pain on each side before and during the extraction. Data were recorded and inserted in SPSS for statistical analysis comparing between the VAS scores between the control and articaine injected sites by means of $\mathrm{t}$ - test and chi square.

Results: A total of 79 patients, 74 patients (93.7\%) of the patients reported no pain sensation " 0 " with both Articaine and Lidocaine. Moreover, 5 patients (6.3\%) of those whom received Articaine reported varying degrees of pain such as: "2", " 4 " and " 5 " were each recorded by $1.3 \%(n=1)$ participants while " 3 " was recorded by $2.5 \%(n=2)$. On the other hand, with lidocaine also $6.3 \%(n=5)$ had varying recordings on the VAS " 1 " and " 4 " were reported by $1.3 \%$ each as for " 2 " it was reported by $3.7 \%(n=3)$. As for the comparison between VAS scores for Lidocaine and Articaine there was no statistical significance between the pain scale scores of Articaine and lidocaine (chi square $p=1$, paired sample t test $p=0.535$ ).

Conclusion: $4 \%$ articaine administrated by means of buccal infiltration only was as efficient as using $2 \%$ lidocaine when administrated by means of conventional infiltration both buccally and palatally.
\end{abstract}

Keywords: Articaine, Lidocaine, Extraction, Maxillary, Buccal, Palatal

\section{Introduction}

Pain is not only an unpleasant sensation, but a complex sensory modality essential for survival. The mechanism by which the nervous system detects a stimulus that has the potential to dam- age the body is important as it triggers behavioral processes that protect against current or further tissue damage [1].

The pain sensation and dentistry goes back to back, especially to those who have poor dental and periodontal health requiring 
surgery, or symptomatic teeth requiring endodontic treatment. Due to the later, patients evaluate a dentist as a good dentist by his ability not to produce little or no discomfort. Administration of local anesthesia intraorally have been highly associated with fear of pain with dentistry, due to the pain associated with the injection and the perceived threat of needle puncture prior to the injection [2].

A Survey carried by Robinson PD., et al. pointed out that most individuals who said to be fearful of dentistry were mostly worried by about receiving injections causing them to delay or miss their appointments [3].

Local anesthetic agents were introduced in 1859 when cocaine was extracted from coca leaves [4]. All anesthetic agents have an intermediate bond making them amides or esters which are linked to an aromatic ring. Therefore, making it lipophilic in order for it to cross the cell membrane. Later on, cocaine was found to be highly addictive and allergic and procaine (cocaine derivative) was introduced as a substitute to cocaine [6]. Lidocaine was first developed by Lofgren in 1943 and it's the first choice of local anesthetic agent in dentistry. This was an amide derivative of diethyl amino acetic acid. The commercially available local anesthetic solution is made of $2 \%$ lidocaine along with 1:80000 epinephrine. With a half-life of 1.5 to 2 hours and a maximum dose of $7 \mathrm{mg} / \mathrm{kg}$ bodyweight if with epinephrine while when available without epinephrine the maximum dose is $4.5 \mathrm{mg} / \mathrm{kg}$ [5].

Articaine was first developed in 1969 as carticaine and was later changed to articaine in 1984 [7].

It has a theophane ring instead of benzene ring [6]. The commercially available for dental use is $4 \%$ solution along with 1:200000 or 1:100000 adrenaline. It also contains a maximum of Na- Salute in $1.0 \mathrm{ml}$ and sodium chloride. Molecular weight is 284 while its half-life is $20 \mathrm{~min}$. its maximum dose is $7 \mathrm{mg} / \mathrm{kg}$ bodyweight. Once its injected into the vascular compartment the unbound local anesthetic is distributed throughout all the body tissue. Due to the theophane ring, its inactivated in the liver as well as hydrolyzation in the tissue and blood [5].

Local anesthetic agents produce anesthesia by inhibiting the excitation mechanism of the nerve endings or by blocking conduction of the peripheral nerves. The mechanism is achieved by inactivating the sodium channels leaving the nerve cells in repolarization phase therefore, the cell won't be excited to achieve the action potential.
As the cell loses its depolarization and the capacity to propagate an impulse, the individual losses the sensation in that area [8]. Maxilla is paired; large maxillary bones extend from the incisive bone rostrally to the nasal bones dorsally. The maxillary bone is fenestrated compact bone that facilitates the spread of local anesthetic agent to reach the dental nerves on the maxilla allowing it to reach the dental nerves and the surrounding periodontium of the maxillary teeth by means of infiltration technique. When a maxillary tooth is to be extracted infiltration technique is used, where you give local anesthesia buccally and palatally to achieve anesthesia of the tooth and the periodontium. The palatal injection is more painful than the buccal injection as the palatal mucosa is closely adherent to the palatal bone of the maxilla, making it the most painful injection sight in the oral cavity [9].

In this study, the aim is to evaluate the use of articaine as buccal infiltration only if sufficient to achieve anesthesia of the maxillary tooth and the periodontium surrounding it without the need of giving palatal infiltration.

\section{Patients and Methods}

A Case Control Study was done Khartoum Teaching Dental Hospital.in a population of Patients attending Khartoum teaching dental hospital for extraction of their maxillary teeth. The study was conducted between November 2019 - January 2020. Inclusion criteria for this study was Patients extracting same permanent maxillary teeth of both sides. The exclusion criteria were patients extracting maxillary teeth from one side only, patients extracting different permanent maxillary teeth of both sides, patients extracting maxillary teeth with abnormal bone or pathology and patients extracting mandibular teeth. The study group was formed from 79 patients whom were clear from any significant systemic disease, attending KDTH outpatient for extraction due to various reasons or referred from orthodontic department to extract upper maxillary teeth on both sides. Articaine 4\% 1:100000 and lidocaine 2\% 1:100000 were used as local anesthetic agent on different sides on different appointments one week apart using different infiltration techniques using sterile dental syringe and a disposable needle 27 gauge $(0.4 \times 21 \mathrm{~mm})$ following aseptic and infection control protocols.

Lidocaine $2 \%$ infiltration technique was as followed, the muccobuccal sulcus and fold were dried near the concerned tooth. 1.75 $\mathrm{mL}$ lidocaine approximately was injected buccally and $0.25 \mathrm{~mL}$ was injected palatally. The extraction was carried out after $5 \mathrm{~min}$; the 
technique was the conventional elevation and forceps technique with mild reflection of palatal gingiva. As for articaine $4 \%$ infiltration technique similar procedure of that of lidocaine was used except no palatal infiltration was done. After the extraction the patients were questioned about the pain intensity on the $100 \mathrm{~mm}$ VAS, descriptors on each end of the scale were NO PAIN and ABSOLUTE PAIN.

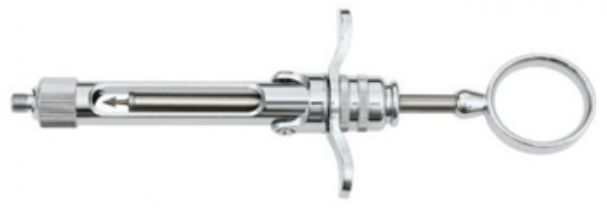

Figure 1: Showing the dental syringe.

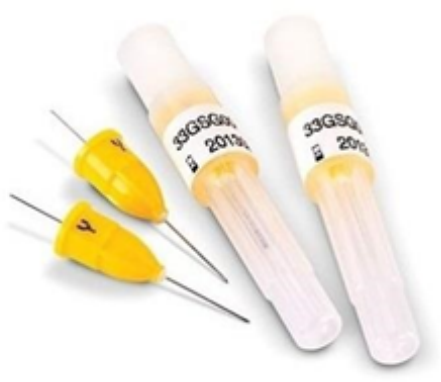

Figure 2: Showing the size 27 short dental needle.

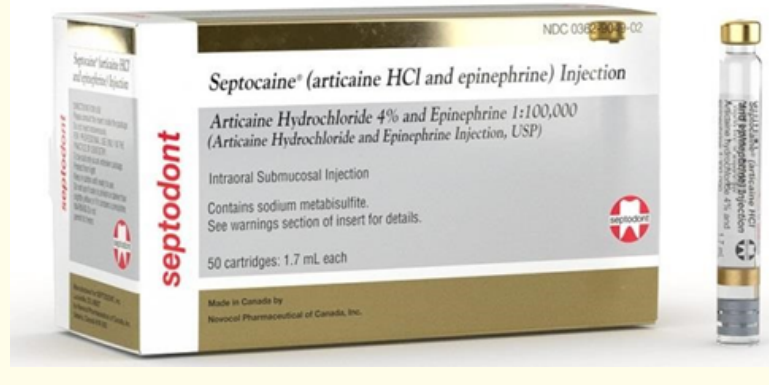

Figure 3: Showing Septocaine ${ }^{\circledR}$ Articaine HCL and epinephrine (Contains 4\% Articaine with 1:100000 epinephrine).

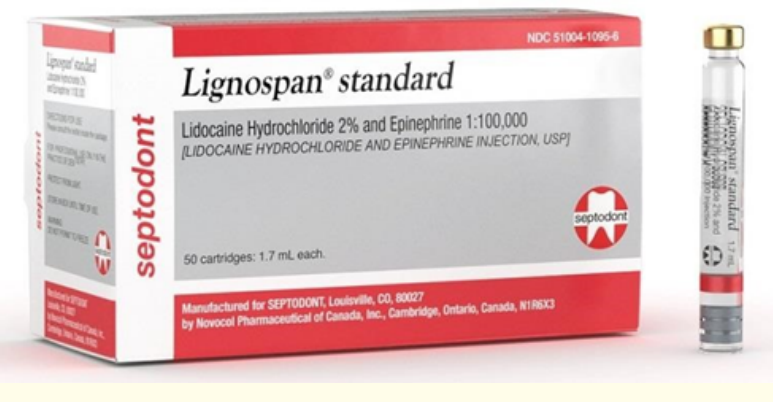

Figure 4: Showing Lignspan ${ }^{\circledR}$ Lidocaine Hydrocholride and epinephrine (Contains 2\% Lidocaine with 1:100000 epinephrine).

The Data collection method and analysis were as follows: Patients with maxillary bilateral extraction were injected using a Dental syringe with a short needle (infiltration) on one side with lidocaine $2 \%$ buccally and palatally (control) and on another appointment the other side was injected with $4 \%$ articaine on the buccal side only (infiltration) using a Dental syringe with a short needle. Visual analogue scale (VAS) was used to assess the pain on each side before and during the extraction. Data will be recorded and inserted in SPSS for statistical analysis to compare between the VAS scores between the control and articaine injected sites by means of $t$ - test and chi square.

\section{Results}

A number of 79 patients attending Khartoum teaching hospital participated in this study where 41 were females and 38 males.

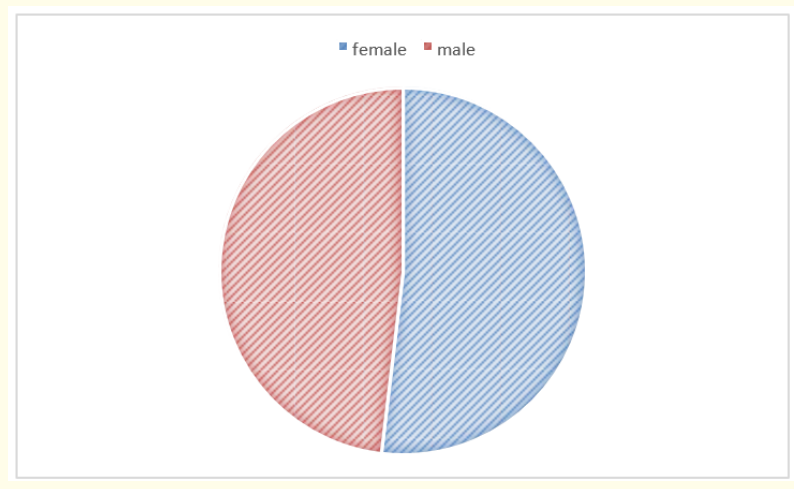

Figure 5: Describing Gender distribution of the patients. 
According to the VAS, $93.7 \%(n=74)$ of the patients reported no pain sensation " 0 " with both Articaine and Lidocaine. Moreover, $6.3 \%(n=5)$ of those whom received Articaine reported varying degrees of pain such as: "2", "4" and "5" were each recorded by $1.3 \%(\mathrm{n}=1)$ participants while " 3 " was recorded by $2.5 \%(\mathrm{n}=2)$. On the other hand, with lidocaine also $6.3 \%(n=5)$ had varying recordings on the VAS " 1 " and " 4 " were reported by $1.3 \%$ each as for "2" it was reported by $3.7 \%(n=3)$. As for the comparison between VAS scores for Lidocaine and Articaine there was no statistical significance between the pain scale scores of Articaine and lidocaine (chi square $p=1$, paired sample t test $p=0.535$ ) concluding that administrating articaine buccally only is as efficient as administrating lidocaine conventionally.

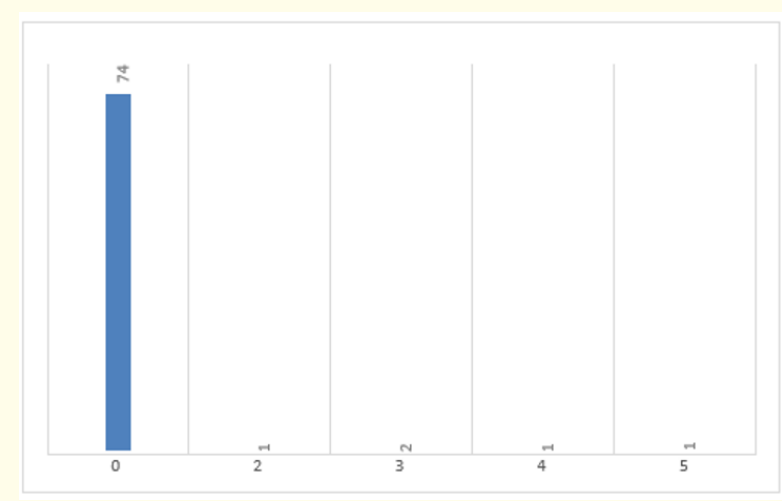

Figure 6: Describes Articaine VAS recorded scores.

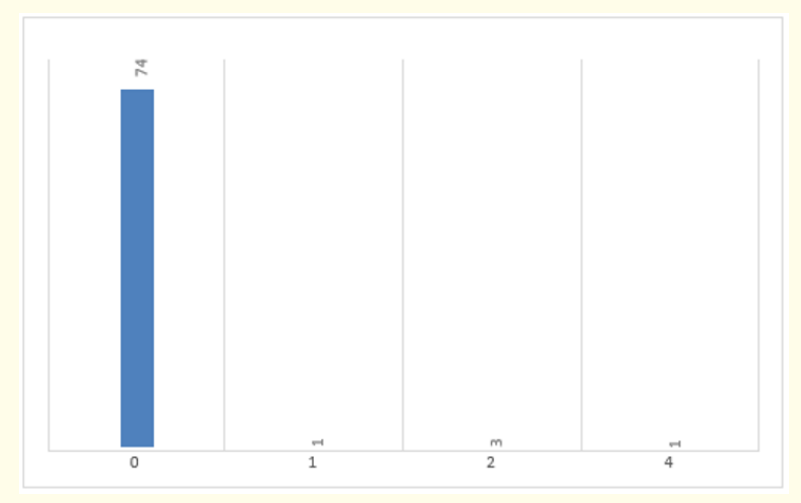

Figure 7: Describes Lidocaine VAS recoded scores.

\begin{tabular}{|c|c|c|c|c|c|}
\hline \multicolumn{2}{|c|}{} & Frequency & Percent & $\begin{array}{c}\text { Valid } \\
\text { Percent }\end{array}$ & $\begin{array}{c}\text { Cumulative } \\
\text { Percent }\end{array}$ \\
\hline Valid & 0 & 74 & 93.7 & 93.7 & 93.7 \\
\cline { 2 - 6 } & 2 & 1 & 1.3 & 1.3 & 94.9 \\
\cline { 2 - 6 } & 3 & 2 & 2.5 & 2.5 & 97.5 \\
\cline { 2 - 6 } & 4 & 1 & 1.3 & 1.3 & 98.7 \\
\cline { 2 - 6 } & 5 & 1 & 1.3 & 1.3 & 100.0 \\
\cline { 2 - 6 } & Total & 79 & 100.0 & 100.0 & \\
\hline
\end{tabular}

Table 1: Describes Articaine VAS recorded scores.

\begin{tabular}{|c|c|c|c|c|c|}
\hline \multicolumn{2}{|c|}{} & Frequency & Percent & $\begin{array}{c}\text { Valid } \\
\text { Percent }\end{array}$ & $\begin{array}{c}\text { Cumulative } \\
\text { Percent }\end{array}$ \\
\hline \multirow{4}{*}{ Valid } & 0 & 74 & 93.7 & 93.7 & 93.7 \\
\cline { 2 - 6 } & 1 & 1 & 1.3 & 1.3 & 94.9 \\
\cline { 2 - 6 } & 2 & 3 & 3.8 & 3.8 & 98.7 \\
\cline { 2 - 6 } & 4 & 1 & 1.3 & 1.3 & 100.0 \\
\cline { 2 - 6 } & Total & 79 & 100.0 & 100.0 & \\
\hline
\end{tabular}

Table 2: Describes Lidocaine VAS recorded scores.

\section{Discussion}

Pain is a protective mechanism like many other body reflexes to protect the body from harm. Several researches been conducted in the field of maxillofacial surgery field to minimize injections and increase the effect of anesthesia [1].

For many years' lidocaine been the leading local anesthetizing agent used for dental surgery. In order to attain sufficient anesthesia in the maxilla lidocaine must be administered in the surgical area both buccally and palatally. Palatal injection itself is painful during the administration of the local anesthetic agent, which is due to the close adherence of the palatal mucosa to the palatal bone. Due to the hydro-separation of the palatal tissue leading to severe pain during the administration. Moreover, the palatal injections cause sialometaplasia (sloughing of the epithelium" caused by ischemia due to the separation of the palatal mucosa from the palatal bone caused by the local anesthetic agent presence between the mucosa and the bone. Patients experience post-operative pain that can be severe at certain times and moreover, the wound might get infected as well with time. Therefore, the use articaine as a local anesthetic agent with buccal infiltration only will allow practitioner to avoid such a complication completely [26].

Articaine's unique amide structure containing a thiophene ring -instead of benzene ring which is a component in lidocaine- and an 
ester group Made it more superior to lidocaine. The thiophene ring allows enables articaine to be more soluble in lipids and increases its potency, allowing greater portion of the administered dose to enter the neurons [27]. The ester group allows the articiane to be hydrolyzed in the plasma therefore having low systemic toxicity making it safe to be used in high concentrations [28]. The long duration of articaine is believed to be due to its high affinity to bind to plasma proteins allowing high concentrations of articaine at the anesthetized area [29].

Results acquired from this study concluded that 4\% Articaine applied by means of buccal infiltration only is as efficient as $2 \%$ Lidocaine applied by means of buccal and palatal infiltration when extracting upper maxillary teeth.

Similar results were acquired by Fan S., et al. (2009) who followed a similar protocol and concluded buccal infiltration only of $4 \%$ Articaine is sufficient to eliminate the pain during the extraction process [14]. Moreover, Dattatrava A., et al. 2014 also compared between the two local anesthetic agents in a similar manner, but less volume of Articaine 0.5 while lidocaine was $0.8 \mathrm{ml}$, nevertheless pain ratings showed no difference between both the groups [16].

Kapol S., et al. 2014 carried a different protocol in his study where both lidocaine and articaine were by means of buccal infiltration only, using $170 \mathrm{~mm}$ Heft Parker visual analogue to assess the pain on the palatal mucosa. It was concluded that articaine was more sufficient lidocaine therefore making articaine more efficient than lidocaine [16]. In the study lidocaine was infiltrated only buccaly hence the patients reported pain this suggests that applying lidocaine buccaly only isn't sufficient to eradicate the pain while articaine does. In the study lidocaine was infiltrated bucaaly and palatally, while articaine was infiltrated palatally only making both lidocaine and articaine sufficient to acquire anesthesia.

Moreover, Omer W., et al. (2018) also reached the conclusion that $4 \%$ articaine whether applied in bucally only or both buccally and palatally patients experienced less pain than the pain felt when $2 \%$ lidocaine was used [18]. Vandana Katyal carried a systemic review to compare the efficacy of and safety of articaine and lidocaine in maxillary and mandibular infiltration and block anesthesia for routine dental treatments. The author concluded that articaine is a superior anesthetic agent than lidocaine [30].
Anwar B., et al. carried a similar study to compare between lidocaine and articaine where he divided the patients into three groups "A: recived lidocaine both palatally and buccally, B: received lidocaine buccally only and C: received articaine buccally only". Statistical significance was found between group A and B and group $A$ and C [26]. Difference in results may be due to the number of participants in the study as our study covered a small sample of participants.

Another study also concluded that $4 \%$ articaine administarted buccally only eradicate the need of lidocaine conventional administration by Daya S., et al. for securing the maxillary arch bar and orthodontic extractions [31].

D Prasanna Kumar compared between 4\% articaine and 2\% lidocaine when administered buccally only to anesthetize the maxillary first molar in order to extract it by means of triple blind randomized controlled study. Out of the articaine group of 50 participants only 6 required an additional injection whereas for lidocaine 29 participants required another injection. Concluding articaine administered bucally only in the maxilla is sufficient to eradicate the pain caused by the tooth extraction process and as efficient as lidocaine when applied conventionally [32].

Articaine's characteristics due to its composition allows it to diffuse better through the fenestrated compact bone of the maxilla through it to reach the palatal mucosa in high concentrations to achieve palatal anesthesia. Haytham al-Mahalawy., et al. conducted a study on New Zealand white rabbits where he measured the concentration of $4 \%$ articaine and that of $2 \%$ lidocaine after administrating them buccally he concluded that articaine had higher concentration in the palatal area than that of lidocaine [33].

\section{Conclusion}

It was concluded in this study that $4 \%$ articaine administrated by means of buccal infiltration only is as efficient as using $2 \%$ lidocaine when administrated by means of conventional infiltration both buccally and palatally. Therefore, making palatal infiltration unnecessary and allowing the operator to avoid causing the patient discomfort and pain.

\section{Recommendation}

Further studies should be carried to study the efficiency of $4 \%$ articaine with regard to gender, age and bone state as well as in- 
creasing the sample size to acquire further solid results, so as to eventually eradicate the use of palatal infiltration technique allowing the patient to have a pleasant visit to the dentist.

\section{Bibliography}

1. Nilesh B. "Guide to pain Management in Low-Resource Settings". International Association for the Study of Pain (2010).

2. Rosenberg ES. "A computer controlled anaesthetic delivery system in periodontal practice: patient satisfaction and acceptance". Journal of Esthetic and Restorative Dentistry 14 (2002): $39-41$.

3. Robinson PD., et al. "Local anaesthesia in dentistry". London: Read Educational and Professional Publishing (2002).

4. Niemann A. "About a new organic base in the coca leaves". Archiv der Pharmazie 153 (1860): 129-155.

5. Rahn R and Ball B. "Local anaesthesia in dentistry - Articaine and Epinephrine for dental anaesthesia”. 1st ed. Seefeld, Germany: 3M ESPE AG (2001).

6. Malamed SF., et al. "Articaine Hydrochloride: a study of the safety of a new amide local anaesthetic". Journal of the American Dental Association 132.2 (2001): 177-185.

7. Malamed SF. "Handbook of local anaesthesia”. 5th ed. St. Louis, Mosby (2004).

8. Fox AJ and Rowbotham DJ. "Anaesthesia". BMJ 319.7209 (1999): 557-60.

9. Tirbod F. "Surgical applied anatomy of the maxillary region". Atlas of Oral Maxillofacial Surgery Clinic 15 (2007): 1-6.

10. Sekhar GR., et al. "Is palatal injection mandatory prior to extraction of maxillary tooth: a preliminary study". Indian Journal of Dental Research 22.1 (2011): 100-102.

11. Yadav S., et al. "Buccal injection of $2 \%$ lidocaine with epinephrine for the removal of maxillary 3rd molars". Anaesthesia Progress 60.3 (2013): 95-98.

12. Lugman U., et al. "Comparison of articaine and lignocaine for uncomplicated exodontia". Journal of College of Physicians and Surgeons Pakistan 25.3 (2015): 181-184.
13. Lima JL., et al. "Comparison of buccal infiltration only of $4 \%$ articaine 1:100000 and 1:200000 epinephrine for extraction of maxillary third molars with pericoronitis: a pilot study". Anaesthesia Progress 60.2 (2013): 42-45.

14. Fan S., et al. "Comparison of the efficiency of permanent maxillary tooth removal performed with single buccal infiltration versus the routine buccal and palatal injection". Oral Surgery, Oral Medicine, Oral Pathology, Oral Radiology, and Endodontology 107.3 (2009): 359-363.

15. Lessemi E., et al. "Anaesthetic efficacy of a labial infiltration method on the nasopalatine nerve". British Dental Journal 205.10 (2008): E21.

16. Dattatrya AD., et al. "A Clinical Study of Efficacy of $4 \%$ Articaine Hydrochloride Versus 2\% Lignocaine Hydrochloride in dentistry". International Oral Health 6.5 (2014): 81-83.

17. Kapol S., et al. "Maxillary posterior removal without palatal injection - Truth or Myth: A dilemma for oral surgeons". Journal of Clinical and Diagnostic Research 8.11 (2014): ZC01-ZC04.

18. Omer W and Aws M. "The Anesthetic Efficacy of Articaine and Lidocaine in Equivalent Doses as Buccal and Non-Palatal Infiltration for Maxillary Molar Extraction: A Randomized, DoubleBlinded, Placebo-Controlled Clinical Trial". Journal of Oral and Maxillofacial Surgery 76.4 (2018): 737-743.

19. Srinivasan N., et al. "Comparison of anesthetic efficiency of $4 \%$ articaine and $2 \%$ lidocaine for maxillary buccal infiltration in patients with irreversible pulpitis". Oral Surgery, Oral Medicine, Oral Pathology, Oral Radiology, and Endodontology 107.1 (2009): 133136.

20. Evans G., et al. "A prospective, randomized, Double blind Comparison of Articaine and Lidocaine for Maxillary Infiltration". Oral Surgery, Oral Medicine, Oral Pathology, Oral Radiology, and Endodontology 34.4 (2008): 389-393.

21. Kanaa MD., et al. "A comparison of the Efficiency of $4 \%$ Articaine 1: 100,000 epinephrine and 2\% Lidocaine 1: 80,000 epinephrine in Achieving Pulpal Anesthesia in Maxillary Teeth with Irreversible Pulpitis". Oral Surgery, Oral Medicine, Oral Pathology, Oral Radiology, and Endodontology 38.3 (2012): 279-282.

22. Costa., et al. "Onset and duration periods of articaine and lidocaine on maxillary infiltration". Quintessence International 36.3 (2005): 197-201. 
23. Kanaa MD., et al. "Articaine and Lidocaine Mandibular Buccal Infiltration Anesthesia: A Prospective Randomized DoubleBlind Cross-Over Study". Journal of Endodontics 32.4 (2006): 296-298.

24. Oliveira PC., et al. "Articaine and lignocaine efficiency in infiltration anesthesia: a pilot study". British Dental Journal 197 (2004): 45-46.

25. David Machine., et al. Sample Size Tables for Clinical Studies. Third edition (2008).

26. Anwar B., et al. "Comparative study of articaine and lidocaine without palatal injection for maxillary teeth extraction". Clinical Oral Investigation 23.8 (2019): 3239-3248.

27. Mathew H and Peter P. "Articaine: A review of the literature". British Dental Journal 210 (2011): 323-329.

28. Ortel R., et al. "Saturable in vitro metabolism of articsine by serum estarses: does it contribute to the persistence of local anesthetic effect?" Reg Anesthesia 21 (1996): 576581.

29. Tucker G T. "Plasma binding and disposition of local anesthetics”. International Anesthesiology Clinics 13 (1975): 33-59.

30. Vandana Katyal. "The efficacy and safety of articaine versus lignocaine in dental treatments: A meta-analysis". Journal of Dentistry 38.4 (2010): 307-317.

31. Daya S., et al. "Effectivness of articaine, single buccal infiltration versus Lidocaine conventional buccal and palatal infiltration in maxillary local anesthesia: A comparative clinical study". Indian Journal of Contemporary Dentistry 7.1 (2019): 47-51.

32. D Prasana Kumar., et al. "Anesthetic efficacy of Single buccal infiltration of $4 \%$ articaine and $2 \%$ lidocaine in extraction of maxillary first Molar". Annals of Maxillofacial Surgery 9.2 (2019): 239-246.

33. Haytham Al-mahalawy., et al. "Articaine Versus Lidocaine Concentration in the Palatal Tissues After Supraperiosteal Buccal Infiltration Anesthesia". Journal of Oral Maxillofacial Surgery 76.2 (2018): 315.e1-315.e7.

\section{Volume 5 Issue 10 October 2021}

(C) All rights are reserved by Hussam Omer Ahmed Mohamed El-Amin and Elneel Ahmed MohamedAli. 